\title{
Weak and Strong Convergence Theorems of Quasi-Nonexpansive Mappings in a Hilbert Spaces
}

\author{
Gang Eun Kim
}

Received: 21 March 2011 / Accepted: 26 August 2011 / Published online: 24 September 2011

(C) The Author(s) 2011. This article is published with open access at Springerlink.com

\begin{abstract}
In this paper, we first prove the weak convergence for the Moudafi's iterative scheme of two quasi-nonexpansive mappings. Then we prove the weak convergence for the Moudafi's iterative scheme of quasi-nonexpansive mapping and nonexpansive mapping. Finally, we prove the strong convergence for the Moudafi's iterative scheme of two quasi-nonexpansive mappings. Our results generalize the recent results due to Iemoto and Takahashi.
\end{abstract}

Keywords Weak and strong convergence $\cdot$ Fixed point · Moudafi iteration process · Quasi-nonexpansive mapping

\section{Introduction}

Approximating fixed points of nonexpansive mappings by an iterative sequence has been investigated by several authors; see, e.g., [1-5] and others. Recently, Iemoto and Takahashi [6] obtained some fundamental properties for nonspreading mappings in a Hilbert space. Further, they studied the approximation of common fixed points of nonexpansive mappings and nonspreading mappings in a Hilbert space by using Moudafi's iterative scheme.

In this paper, we first prove the weak convergence of two quasi-nonexpansive mappings in a Hilbert space by using Moudafi's iterative scheme. Then we prove the weak convergence of quasi-nonexpansive mapping and nonexpansive mapping in a Hilbert space by using Moudafi's iterative scheme. Finally, we prove the strong convergence of two quasi-nonexpansive mappings satisfying condition $\mathbf{A}$ in a Hilbert

Communicated by Viorel Barbu.

G.E. Kim $(\bowtie)$

Department of Applied Mathematics, Pukyong National University, Busan 608-737, Korea

e-mail: kimge@pknu.ac.kr 
space by using Moudafi's iterative scheme. Our results generalize the recent results due to Iemoto and Takahashi [6].

\section{Preliminaries}

Throughout this paper, we denote by $H$ a real Hilbert space. Let $C$ be a nonempty, closed, and convex subset of $H$. Then a mapping $T$ of $C$ into itself is called nonexpansive iff $\|T x-T y\| \leq\|x-y\|$ for all $x, y \in C$. We denote by $F(T)$ the set of all fixed points of $T$, i.e., $F(T):=\{x \in C: T x=x\}$. A mapping $T$ from $C$ into $C$ is also called quasi-nonexpansive iff the set $F(T)$ of fixed points of $T$ is nonempty and $\|T x-y\| \leq\|x-y\|$ for all $x \in C$ and $y \in F(T)$. For two mappings $S, T$ of $C$ into itself, we consider the following iteration scheme (Moudafi [7]): $x_{1} \in C$,

$$
x_{n+1}=\left(1-\alpha_{n}\right) x_{n}+\alpha_{n}\left[\beta_{n} S x_{n}+\left(1-\beta_{n}\right) T x_{n}\right]
$$

for all $n \geq 1$, where $\left\{\alpha_{n}\right\}$ and $\left\{\beta_{n}\right\}$ are real sequences in $[0,1]$. If $\beta_{n}=0$, then such an iteration scheme shrinks to that introduced by Mann [1]. In a Hilbert space, it is known that

$$
\|\alpha x+(1-\alpha) y\|^{2}=\alpha\|x\|^{2}+(1-\alpha)\|y\|^{2}-\alpha(1-\alpha)\|x-y\|^{2}
$$

for all $x, y \in H$ and $\alpha \in \mathbb{R}$; see [8]. A Banach space $E$ is called uniformly convex iff for each $\epsilon>0$ there is a $\delta>0$ such that for $x, y \in E$ with $\|x\|,\|y\| \leq 1$ and $\|x-y\| \geq$ $\epsilon,\|x+y\| \leq 2(1-\delta)$ holds. Let $E$ be a smooth, strictly convex and reflexive Banach space, let $J$ be the duality mapping of $E$ and let $C$ be a nonempty, closed, and convex subset of $E$. Then a mapping $T: C \rightarrow C$ is said to be nonspreading [6] iff

$$
\phi(T x, T y)+\phi(T y, T x) \leq \phi(T x, y)+\phi(T y, x)
$$

for all $x, y \in C$, where $\phi(x, y):=\|x\|^{2}-2\langle x, J(y)\rangle+\|y\|^{2}$ for all $x, y \in E$. In the case when $E$ is a Hilbert space, we know that $\phi(x, y)=\|x-y\|^{2}$ for all $x, y \in E$. So, a nonspreading mapping $T: C \rightarrow C$ in a Hilbert space $H$ is defined as follows:

$$
2\|T x-T y\|^{2} \leq\|T x-y\|^{2}+\|x-T y\|^{2}
$$

for all $x, y \in C$. Thus, Iemoto and Takahashi [6] proved that it is equivalent to

$$
\|T x-T y\|^{2} \leq\|x-y\|^{2}+2\langle x-T x, y-T y\rangle
$$

for all $x, y \in C$. We know that in a Hilbert space, if the set of fixed points of a nonspreading mapping is nonempty, then the nonspreading mapping is quasinonexpansive; see [6]. When $\left\{x_{n}\right\}$ is a sequence in $E$, then $x_{n} \rightarrow x\left(x_{n} \rightarrow x\right)$ will denote strong (weak) convergence of the sequence $\left\{x_{n}\right\}$ to $x$. A mapping $T: C \rightarrow H$ is said to be demiclosed at $y \in H$ [9] iff for any sequence $\left\{x_{n}\right\}$ in $C$, it follows from $x_{n} \rightarrow x$ and $T x_{n} \rightarrow y$ that $x \in C$ and $T(x)=y$. If $I-T$ is demiclosed at zero, i.e., for any sequence $\left\{x_{n}\right\}$ in $C$, the conditions $x_{n} \rightarrow x$ and $x_{n}-T x_{n} \rightarrow 0$ imply $x-T x=0$. 
We know that $H$ satisfies Opial's condition [10], that is, for any sequence $\left\{x_{n}\right\}$ in $H$, $x_{n} \rightarrow x$ implies that

$$
\limsup _{n \rightarrow \infty}\left\|x_{n}-x\right\|<\limsup _{n \rightarrow \infty}\left\|x_{n}-y\right\|
$$

for all $y \in H$ with $y \neq x$. All Hilbert spaces and $l^{p}(1<p<\infty)$ satisfy Opial's condition, while $L^{p}$ with $1<p \neq 2<\infty$ do not. Two mappings $S, T: C \rightarrow C$, where $C$ is a subset of $E$, are said to satisfy condition $\mathbf{A}$ [11] (cf. [12]) iff there exists a nondecreasing function $f:[0, \infty] \rightarrow[0, \infty]$ with $f(0)=0$ and $f(r)>0$ for all $r \in[0, \infty]$ such that

$$
\frac{1}{2}(\|x-T x\|+\|x-S x\|) \geq f(d(x, \mathbf{F}))
$$

for all $x \in C$, where $\mathbf{F}=F(S) \cap F(T) \neq \emptyset$ and $d(x, \mathbf{F})=\inf _{z \in \mathbf{F}}\|x-z\|$.

\section{Weak and Strong Convergence Theorems}

We first begin with the following lemma.

Lemma 3.1 [4] Let $\left\{a_{n}\right\}$ and $\left\{b_{n}\right\}$ be sequences of nonnegative real numbers such that $\sum_{n=1}^{\infty} b_{n}<\infty$ and

$$
a_{n+1} \leq a_{n}+b_{n}
$$

for all $n \geq 1$. Then $\lim _{n \rightarrow \infty} a_{n}$ exists.

Lemma 3.2 [13] Let $E$ be a uniformly convex Banach space. Let $x, y \in E$. If $\|x\| \leq$ $1,\|y\| \leq 1$, and $\|x-y\| \geq \epsilon>0$, then $\|\lambda x+(1-\lambda) y\| \leq 1-2 \lambda(1-\lambda) \delta(\epsilon)$ for $\lambda$ with $0 \leq \lambda \leq 1$.

Our Theorem 3.1 carries over Theorem 4.1(i), (iii) of Iemoto and Takahashi [6] to quasi-nonexpansive mappings.

Theorem 3.1 Let $H$ be a Hilbert space and $C$ be a nonempty, closed and convex subset of $H$, and let $S, T$ be two quasi-nonexpansive mappings of $C$ into itself such that $I-T, I-S$ are demiclosed at zero with $F(S) \cap F(T) \neq \emptyset$. Suppose that, for any $x_{1}$ in $C,\left\{x_{n}\right\}$ is defined by (1). Then the following hold:

(i) If $\liminf _{n \rightarrow \infty} \alpha_{n}\left(1-\alpha_{n}\right)>0$ and $\sum_{n=1}^{\infty}\left(1-\beta_{n}\right)<\infty$, then $\left\{x_{n}\right\}$ converges weakly to $p \in F(S)$

(ii) if $\liminf _{n \rightarrow \infty} \alpha_{n}\left(1-\alpha_{n}\right)>0$ and $\liminf _{n \rightarrow \infty} \beta_{n}\left(1-\beta_{n}\right)>0$, then $\left\{x_{n}\right\}$ converges weakly to $p \in F(S) \cap F(T)$.

Proof For any $z \in F(S) \cap F(T)$, since

$$
\left\|x_{n+1}-z\right\|=\left\|\left(1-\alpha_{n}\right) x_{n}+\alpha_{n}\left[\beta_{n} S x_{n}+\left(1-\beta_{n}\right) T x_{n}\right]-z\right\|
$$




$$
\begin{aligned}
& \leq\left(1-\alpha_{n}\right)\left\|x_{n}-z\right\|+\alpha_{n}\left\|\left[\beta_{n} S x_{n}+\left(1-\beta_{n}\right) T x_{n}\right]-z\right\| \\
& \leq\left(1-\alpha_{n}\right)\left\|x_{n}-z\right\|+\alpha_{n}\left\{\beta_{n}\left\|S x_{n}-z\right\|+\left(1-\beta_{n}\right)\left\|T x_{n}-z\right\|\right\} \\
& \leq\left(1-\alpha_{n}\right)\left\|x_{n}-z\right\|+\alpha_{n}\left\{\beta_{n}\left\|x_{n}-z\right\|+\left(1-\beta_{n}\right)\left\|x_{n}-z\right\|\right\} \\
& =\left\|x_{n}-z\right\|
\end{aligned}
$$

for all $n \geq 1$. We readily see that

$$
\lim _{n \rightarrow \infty}\left\|x_{n}-z\right\|(\equiv c)
$$

exists, and thus $\left\{x_{n}\right\}$ is bounded. (i) put $z_{n+1}=\left(1-\alpha_{n}\right) x_{n}+\alpha_{n} S x_{n}$. Then we obtain

$$
\begin{aligned}
\left\|x_{n+1}-z_{n+1}\right\| & =\left\|\left(1-\alpha_{n}\right) x_{n}+\alpha_{n}\left[\beta_{n} S x_{n}+\left(1-\beta_{n}\right) T x_{n}\right]-\left(1-\alpha_{n}\right) x_{n}-\alpha_{n} S x_{n}\right\| \\
& =\alpha_{n}\left\|\beta_{n} S x_{n}+\left(1-\beta_{n}\right) T x_{n}-S x_{n}\right\| \\
& =\alpha_{n}\left(1-\beta_{n}\right)\left\|T x_{n}-S x_{n}\right\| \\
& \leq\left(1-\beta_{n}\right)\left\|T x_{n}-S x_{n}\right\|,
\end{aligned}
$$

where $\sup _{n \geq 1}\left\|T x_{n}-S x_{n}\right\|<\infty$. Since $\sum_{n=1}^{\infty}\left(1-\beta_{n}\right)<\infty$, we obtain $\left\|x_{n}-z_{n}\right\| \rightarrow$ 0 as $n \rightarrow \infty$ and thus

$$
\lim _{n \rightarrow \infty}\left\|z_{n}-z\right\|=\lim _{n \rightarrow \infty}\left\|x_{n}-z\right\|=c
$$

From (2), we obtain

$$
\begin{aligned}
\left\|z_{n+1}-z\right\|^{2} & =\left\|\left(1-\alpha_{n}\right) x_{n}+\alpha_{n} S x_{n}-z\right\|^{2} \\
& =\left\|\left(1-\alpha_{n}\right)\left(x_{n}-z\right)+\alpha_{n}\left(S x_{n}-z\right)\right\|^{2} \\
& =\left(1-\alpha_{n}\right)\left\|x_{n}-z\right\|^{2}+\alpha_{n}\left\|S x_{n}-z\right\|^{2}-\alpha_{n}\left(1-\alpha_{n}\right)\left\|x_{n}-S x_{n}\right\|^{2} \\
& \leq\left(1-\alpha_{n}\right)\left\|x_{n}-z\right\|^{2}+\alpha_{n}\left\|x_{n}-z\right\|^{2}-\alpha_{n}\left(1-\alpha_{n}\right)\left\|x_{n}-S x_{n}\right\|^{2} \\
& =\left\|x_{n}-z\right\|^{2}-\alpha_{n}\left(1-\alpha_{n}\right)\left\|x_{n}-S x_{n}\right\|^{2} .
\end{aligned}
$$

Thus,

$$
\alpha_{n}\left(1-\alpha_{n}\right)\left\|x_{n}-S x_{n}\right\|^{2} \leq\left\|x_{n}-z\right\|^{2}-\left\|z_{n+1}-z\right\|^{2} .
$$

Since $\liminf _{n \rightarrow \infty} \alpha_{n}\left(1-\alpha_{n}\right)>0$ and by (4), we obtain

$$
\lim _{n \rightarrow \infty}\left\|S x_{n}-x_{n}\right\|=0 .
$$

Since $\left\{x_{n}\right\}$ is a bounded sequence, there exist the subsequences $\left\{x_{n_{i}}\right\}$ and $\left\{x_{n_{j}}\right\}$ of $\left\{x_{n}\right\}$ such that $\left\{x_{n_{i}}\right\} \rightarrow p$ and $\left\{x_{n_{j}}\right\} \rightarrow q$, respectively. Since $I-S$ is demiclosed at zero and by (5), we have $p, q \in F(S)$. We first show that for any $y \in F(S), \lim _{n \rightarrow \infty} \| x_{n}-$ $y \|$ exists. In fact, for any $y \in F(S)$,

$$
\left\|z_{n+1}-y\right\|=\left\|\left(1-\alpha_{n}\right) x_{n}+\alpha_{n} S x_{n}-y\right\|
$$




$$
\begin{aligned}
& \leq\left(1-\alpha_{n}\right)\left\|x_{n}-y\right\|+\alpha_{n}\left\|S x_{n}-y\right\| \\
& \leq\left\|x_{n}-y\right\| \\
& \leq\left\|z_{n}-y\right\|+\left\|x_{n}-z_{n}\right\| .
\end{aligned}
$$

By Lemma 3.1, $\lim _{n \rightarrow \infty}\left\|z_{n}-y\right\|$ exists. So, we see that $\lim _{n \rightarrow \infty}\left\|x_{n}-y\right\|$ exists by $x_{n}-z_{n} \rightarrow 0$ as $n \rightarrow \infty$. Next, we show $p=q$. If not, by Opial's condition,

$$
\begin{aligned}
\lim _{n \rightarrow \infty}\left\|x_{n}-p\right\| & =\lim _{i \rightarrow \infty}\left\|x_{n_{i}}-p\right\| \\
& <\lim _{i \rightarrow \infty}\left\|x_{n_{i}}-q\right\| \\
& =\lim _{n \rightarrow \infty}\left\|x_{n}-q\right\| \\
& =\lim _{j \rightarrow \infty}\left\|x_{n_{j}}-q\right\| \\
& <\lim _{j \rightarrow \infty}\left\|x_{n_{j}}-p\right\| \\
& =\lim _{n \rightarrow \infty}\left\|x_{n}-p\right\| .
\end{aligned}
$$

This is a contradiction. Hence, we have $p=q$. Hence, $\left\{x_{n}\right\}$ converges weakly to $p \in F(S)$.

(ii) By using (1), we obtain

$$
x_{n+1}=\beta_{n}\left[\left(1-\alpha_{n}\right) x_{n}+\alpha_{n} S x_{n}\right]+\left(1-\beta_{n}\right)\left[\left(1-\alpha_{n}\right) x_{n}+\alpha_{n} T x_{n}\right]
$$

for all $n \geq 1$. Putting $V_{n}=\beta_{n}\left[\left(1-\alpha_{n}\right) I+\alpha_{n} S\right]+\left(1-\beta_{n}\right)\left[\left(1-\alpha_{n}\right) I+\alpha_{n} T\right]$. Then $x_{n+1}=\bar{V}_{n} x_{n}$. For any $z \in F(S) \cap F(T)$,

$$
\begin{aligned}
\left\|V_{n} x_{n}-z\right\| & =\left\|\beta_{n}\left[\left(1-\alpha_{n}\right) x_{n}+\alpha_{n} S x_{n}\right]+\left(1-\beta_{n}\right)\left[\left(1-\alpha_{n}\right) x_{n}+\alpha_{n} T x_{n}\right]-z\right\| \\
& \leq \beta_{n}\left\|\left(1-\alpha_{n}\right) x_{n}+\alpha_{n} S x_{n}-z\right\|+\left(1-\beta_{n}\right)\left\|\left(1-\alpha_{n}\right) x_{n}+\alpha_{n} T x_{n}-z\right\| \\
& \leq \beta_{n}\left\|\left(1-\alpha_{n}\right) x_{n}+\alpha_{n} S x_{n}-z\right\|+\left(1-\beta_{n}\right)\left\|x_{n}-z\right\| \\
& \leq\left\|x_{n}-z\right\|
\end{aligned}
$$

and thus

$$
\begin{aligned}
0 & \leq\left\|x_{n}-z\right\|-\beta_{n}\left\|\left(1-\alpha_{n}\right) x_{n}+\alpha_{n} S x_{n}-z\right\|-\left(1-\beta_{n}\right)\left\|x_{n}-z\right\| \\
& =\beta_{n}\left(\left\|x_{n}-z\right\|-\left\|\left(1-\alpha_{n}\right) x_{n}+\alpha_{n} S x_{n}-z\right\|\right) \\
& \leq\left\|x_{n}-z\right\|-\left\|V_{n} x_{n}-z\right\| .
\end{aligned}
$$

By using (3),

$$
0 \leq\left\|x_{n}-z\right\|-\left\|V_{n} x_{n}-z\right\|=\left\|x_{n}-z\right\|-\left\|x_{n+1}-z\right\| \rightarrow c-c=0
$$

as $n \rightarrow \infty$. So, we obtain

$$
\begin{aligned}
0 & \leq\left(1-\beta_{n}\right) \beta_{n}\left(\left\|x_{n}-z\right\|-\left\|\left(1-\alpha_{n}\right) x_{n}+\alpha_{n} S x_{n}-z\right\|\right) \\
& \leq\left(1-\beta_{n}\right)\left(\left\|x_{n}-z\right\|-\left\|V_{n} x_{n}-z\right\|\right) .
\end{aligned}
$$


Since $\liminf _{n \rightarrow \infty} \beta_{n}\left(1-\beta_{n}\right)>0$, it follows from (6) that

$$
\lim _{n \rightarrow \infty}\left(\left\|x_{n}-z\right\|-\left\|\left(1-\alpha_{n}\right) x_{n}+\alpha_{n} S x_{n}-z\right\|\right)=0 .
$$

Since

$$
\left\|S x_{n}-z\right\| \leq\left\|x_{n}-z\right\|
$$

and by Lemma 3.2 and Takahashi [14], we have

$$
\begin{aligned}
& \left\|\left(1-\alpha_{n}\right) x_{n}+\alpha_{n} S x_{n}-z\right\| \\
& \quad=\left\|\left(1-\alpha_{n}\right)\left(x_{n}-z\right)+\alpha_{n}\left(S x_{n}-z\right)\right\| \\
& \quad \leq\left\|x_{n}-z\right\|\left[1-2 \alpha_{n}\left(1-\alpha_{n}\right) \delta_{E}\left(\frac{\left\|S x_{n}-x_{n}\right\|}{\left\|x_{n}-z\right\|}\right)\right] .
\end{aligned}
$$

Hence, we obtain

$$
2 \alpha_{n}\left(1-\alpha_{n}\right)\left\|x_{n}-z\right\| \delta_{E}\left(\frac{\left\|S x_{n}-x_{n}\right\|}{\left\|x_{n}-z\right\|}\right) \leq\left\|x_{n}-z\right\|-\left\|\left(1-\alpha_{n}\right) x_{n}+\alpha_{n} S x_{n}-z\right\| .
$$

Since $\delta_{E}$ is strictly increasing, continuous, and by $\liminf _{n \rightarrow \infty} \alpha_{n}\left(1-\alpha_{n}\right)>0$ and (7), we obtain

$$
\lim _{n \rightarrow \infty}\left\|S x_{n}-x_{n}\right\|=0 .
$$

Since $I-S$ is demiclosed at zero and by using the same method in the proof of (i), we obtain that if $x_{n_{i}} \rightarrow p$, then $p \in F(S)$. We also prove that $p \in F(T)$. In fact, we obtain that for any $z \in F(S) \cap F(T)$,

$$
\begin{aligned}
\left\|V_{n} x_{n}-z\right\| & =\left\|\beta_{n}\left[\left(1-\alpha_{n}\right) x_{n}+\alpha_{n} S x_{n}\right]+\left(1-\beta_{n}\right)\left[\left(1-\alpha_{n}\right) x_{n}+\alpha_{n} T x_{n}\right]-z\right\| \\
& \leq \beta_{n}\left\|\left(1-\alpha_{n}\right) x_{n}+\alpha_{n} S x_{n}-z\right\|+\left(1-\beta_{n}\right)\left\|\left(1-\alpha_{n}\right) x_{n}+\alpha_{n} T x_{n}-z\right\| \\
& \leq \beta_{n}\left\|x_{n}-z\right\|+\left(1-\beta_{n}\right)\left\|\left(1-\alpha_{n}\right) x_{n}+\alpha_{n} T x_{n}-z\right\| \\
& \leq\left\|x_{n}-z\right\|
\end{aligned}
$$

and hence

$$
\begin{aligned}
0 & \leq\left(1-\beta_{n}\right)\left(\left\|x_{n}-z\right\|-\left\|\left(1-\alpha_{n}\right) x_{n}+\alpha_{n} T x_{n}-z\right\|\right) \\
& \leq\left\|x_{n}-z\right\|-\left\|V_{n} x_{n}-z\right\| .
\end{aligned}
$$

So, we have

$$
\begin{aligned}
0 & \leq \beta_{n}\left(1-\beta_{n}\right)\left(\left\|x_{n}-z\right\|-\left\|\left(1-\alpha_{n}\right) x_{n}+\alpha_{n} T x_{n}-z\right\|\right) \\
& \leq \beta_{n}\left(\left\|x_{n}-z\right\|-\left\|V_{n} x_{n}-z\right\|\right) .
\end{aligned}
$$

Since $\liminf _{n \rightarrow \infty} \beta_{n}\left(1-\beta_{n}\right)>0$, it follows from (6) that

$$
\lim _{n \rightarrow \infty}\left(\left\|x_{n}-z\right\|-\left\|\left(1-\alpha_{n}\right) x_{n}+\alpha_{n} T x_{n}-z\right\|\right)=0 .
$$


Since

$$
\left\|T x_{n}-z\right\| \leq\left\|x_{n}-z\right\|
$$

and by Lemma 3.2 and Takahashi [14], we have

$$
\begin{aligned}
& \left\|\left(1-\alpha_{n}\right) x_{n}+\alpha_{n} T x_{n}-z\right\| \\
& \quad=\left\|\left(1-\alpha_{n}\right)\left(x_{n}-z\right)+\alpha_{n}\left(T x_{n}-z\right)\right\| \\
& \quad \leq\left\|x_{n}-z\right\|\left[1-2 \alpha_{n}\left(1-\alpha_{n}\right) \delta_{E}\left(\frac{\left\|T x_{n}-x_{n}\right\|}{\left\|x_{n}-z\right\|}\right)\right] .
\end{aligned}
$$

Hence, we obtain

$$
2 \alpha_{n}\left(1-\alpha_{n}\right)\left\|x_{n}-z\right\| \delta_{E}\left(\frac{\left\|T x_{n}-x_{n}\right\|}{\left\|x_{n}-z\right\|}\right) \leq\left\|x_{n}-z\right\|-\left\|\left(1-\alpha_{n}\right) x_{n}+\alpha_{n} T x_{n}-z\right\| .
$$

Since $\delta_{E}$ is strictly increasing, continuous, and by $\liminf _{n \rightarrow \infty} \alpha_{n}\left(1-\alpha_{n}\right)>0$ and (8), we obtain

$$
\lim _{n \rightarrow \infty}\left\|T x_{n}-x_{n}\right\|=0
$$

Since $\left\{x_{n_{i}}\right\} \rightarrow p$ and $I-T$ is demiclosed at zero, then $p \in F(T)$. Let $\left\{x_{n_{j}}\right\}$ be another subsequence of $\left\{x_{n}\right\}$ such that $\left\{x_{n_{j}}\right\} \rightarrow q$. Then we obtain $p=q$. If not, by Opial's condition and (3),

$$
\begin{aligned}
\lim _{n \rightarrow \infty}\left\|x_{n}-p\right\| & =\lim _{i \rightarrow \infty}\left\|x_{n_{i}}-p\right\| \\
& <\lim _{i \rightarrow \infty}\left\|x_{n_{i}}-q\right\| \\
& =\lim _{n \rightarrow \infty}\left\|x_{n}-q\right\|
\end{aligned}
$$

and by using similar method, we have

$$
\lim _{n \rightarrow \infty}\left\|x_{n}-q\right\|<\lim _{n \rightarrow \infty}\left\|x_{n}-p\right\|
$$

This is a contradiction. Hence, we have $p=q$. Therefore, $\left\{x_{n}\right\}$ converges weakly to $p \in F(S) \cap F(T)$.

Our Theorem 3.2 carries over Theorem 4.1(ii) of Iemoto and Takahashi [6] to a quasi-nonexpansive mapping.

Theorem 3.2 Let $H$ be a Hilbert space and $C$ be a nonempty, closed, and convex subset of $H$, and let $S$ be a quasi-nonexpansive mapping of $C$ into itself and $T$ be a nonexpansive mapping of $C$ into itself such that $F(S) \cap F(T) \neq \emptyset$. Suppose that, for any $x_{1}$ in $C,\left\{x_{n}\right\}$ is defined by (1), where $\left\{\alpha_{n}\right\}$ and $\left\{\beta_{n}\right\}$ are chosen so that $\sum_{n=1}^{\infty} \alpha_{n}\left(1-\alpha_{n}\right)=\infty$ and $\sum_{n=1}^{\infty} \beta_{n}<\infty$. Then $\left\{x_{n}\right\}$ converges weakly to $p \in F(T)$. 
Proof For any $z \in F(S) \cap F(T)$. Put $z_{n+1}=\left(1-\alpha_{n}\right) x_{n}+\alpha_{n} T x_{n}$. Then

$$
\begin{aligned}
\left\|x_{n+1}-z_{n+1}\right\|= & \|\left(1-\alpha_{n}\right) x_{n}+\alpha_{n}\left[\beta_{n} S x_{n}+\left(1-\beta_{n}\right) T x_{n}\right] \\
& -\left(1-\alpha_{n}\right) x_{n}-\alpha_{n} T x_{n} \| \\
= & \alpha_{n}\left\|\beta_{n} S x_{n}+\left(1-\beta_{n}\right) T x_{n}-T x_{n}\right\| \\
= & \alpha_{n} \beta_{n}\left\|T x_{n}-S x_{n}\right\| \\
\leq & \beta_{n} M
\end{aligned}
$$

where $M=\sup _{n \geq 1}\left\|T x_{n}-S x_{n}\right\|<\infty$. Since $\sum_{n=1}^{\infty} \beta_{n}<\infty$, we obtain $\left\|x_{n}-z_{n}\right\| \rightarrow$ 0 as $n \rightarrow \infty$. Since

$$
\begin{aligned}
\left\|z_{n+1}-z\right\| & =\left\|\left(1-\alpha_{n}\right) x_{n}+\alpha_{n} T x_{n}-z\right\| \\
& \leq\left(1-\alpha_{n}\right)\left\|x_{n}-z\right\|+\alpha_{n}\left\|T x_{n}-z\right\| \\
& \leq\left\|x_{n}-z\right\| \\
& \leq\left\|z_{n}-z\right\|+\left\|x_{n}-z_{n}\right\|,
\end{aligned}
$$

and by Lemma 3.1, $\lim _{n \rightarrow \infty}\left\|z_{n}-z\right\|$ exists. So, we see that $\lim _{n \rightarrow \infty}\left\|x_{n}-z\right\|$ exists by $x_{n}-z_{n} \rightarrow 0$ as $n \rightarrow \infty$, and thus $\left\{x_{n}\right\}$ is bounded. By using (9), we obtain

$$
\begin{aligned}
\left\|x_{n+1}-z\right\| & \leq\left\|x_{n+1}-z_{n+1}\right\|+\left\|z_{n+1}-z\right\| \\
& \leq \beta_{n} M+\left\|z_{n+1}-z\right\|
\end{aligned}
$$

and thus

$$
\left\|x_{n+1}-z\right\|-\beta_{n} M \leq\left\|z_{n+1}-z\right\| \text {. }
$$

Since

$$
\left\|T x_{n}-z\right\| \leq\left\|x_{n}-z\right\|
$$

and by Lemma 3.2 and Takahashi [14], we have

$$
\begin{aligned}
\left\|z_{n+1}-z\right\| & =\left\|\left(1-\alpha_{n}\right) x_{n}+\alpha_{n} T x_{n}-z\right\| \\
& =\left\|\left(1-\alpha_{n}\right)\left(x_{n}-z\right)+\alpha_{n}\left(T x_{n}-z\right)\right\| \\
& \leq\left\|x_{n}-z\right\|\left[1-2 \alpha_{n}\left(1-\alpha_{n}\right) \delta_{E}\left(\frac{\left\|T x_{n}-x_{n}\right\|}{\left\|x_{n}-z\right\|}\right)\right] .
\end{aligned}
$$

Hence, by (10), we obtain

$$
\begin{aligned}
2 \alpha_{n}\left(1-\alpha_{n}\right)\left\|x_{n}-z\right\| \delta_{E}\left(\frac{\left\|T x_{n}-x_{n}\right\|}{\left\|x_{n}-z\right\|}\right) & \leq\left\|x_{n}-z\right\|-\left\|z_{n+1}-z\right\| \\
& \leq\left\|x_{n}-z\right\|-\left\|x_{n+1}-z\right\|+\beta_{n} M .
\end{aligned}
$$


Since $\delta_{E}$ is strictly increasing, continuous, $\sum_{n=1}^{\infty} \alpha_{n}\left(1-\alpha_{n}\right)=\infty$ and $\sum_{n=1}^{\infty} \beta_{n}<$ $\infty$, we obtain

$$
\liminf _{n \rightarrow \infty}\left\|T x_{n}-x_{n}\right\|=0 .
$$

We note that $T x_{n+1}-x_{n+1}=T x_{n+1}-U_{n} x_{n}+\left(1-\alpha_{n}\right)\left(U_{n} x_{n}-x_{n}\right)$, where $U_{n}=$ $\beta_{n} S+\left(1-\beta_{n}\right) T$. Then we have that

$$
\begin{aligned}
\left\|x_{n+1}-T x_{n+1}\right\| \\
\quad \leq\left\|T x_{n+1}-T x_{n}\right\|+\left\|T x_{n}-U_{n} x_{n}\right\|+\left(1-\alpha_{n}\right)\left\|U_{n} x_{n}-x_{n}\right\| \\
\quad \leq\left\|x_{n+1}-x_{n}\right\|+\left\|T x_{n}-U_{n} x_{n}\right\|+\left(1-\alpha_{n}\right)\left\|U_{n} x_{n}-x_{n}\right\| \\
\quad=\left\|\left(1-\alpha_{n}\right) x_{n}+\alpha_{n} U_{n} x_{n}-x_{n}\right\|+\left\|T x_{n}-U_{n} x_{n}\right\|+\left(1-\alpha_{n}\right)\left\|U_{n} x_{n}-x_{n}\right\| \\
\quad=\alpha_{n}\left\|U_{n} x_{n}-x_{n}\right\|+\left\|T x_{n}-U_{n} x_{n}\right\|+\left(1-\alpha_{n}\right)\left\|U_{n} x_{n}-x_{n}\right\| \\
=\left\|U_{n} x_{n}-x_{n}\right\|+\left\|T x_{n}-\left(\beta_{n} S x_{n}+\left(1-\beta_{n}\right) T x_{n}\right)\right\| \\
\quad=\left\|\beta_{n}\left(S x_{n}-x_{n}\right)+\left(1-\beta_{n}\right)\left(T x_{n}-x_{n}\right)\right\|+\beta_{n}\left\|T x_{n}-S x_{n}\right\| \\
\quad \leq\left(1-\beta_{n}\right)\left\|x_{n}-T x_{n}\right\|+\beta_{n}\left(\left\|x_{n}-S x_{n}\right\|+\left\|T x_{n}-S x_{n}\right\|\right) \\
\quad \leq\left(1-\beta_{n}\right)\left\|x_{n}-T x_{n}\right\|+\beta_{n}\left(\left\|x_{n}-T x_{n}\right\|+\left\|T x_{n}-S x_{n}\right\|+\left\|T x_{n}-S x_{n}\right\|\right) \\
\quad \leq\left\|x_{n}-T x_{n}\right\|+2 \beta_{n}\left\|T x_{n}-S x_{n}\right\| \\
\quad \leq\left\|x_{n}-T x_{n}\right\|+2 \beta_{n} M .
\end{aligned}
$$

Since $\sum_{n=1}^{\infty} \beta_{n}<\infty$ and by Lemma $3.1, \lim _{n \rightarrow \infty}\left\|x_{n}-T x_{n}\right\|$ exists. Hence, by (11), we obtain

$$
\lim _{n \rightarrow \infty}\left\|T x_{n}-x_{n}\right\|=0
$$

Since $\left\{x_{n}\right\}$ is bounded, there exist the subsequences $\left\{x_{n_{i}}\right\}$ and $\left\{x_{n_{j}}\right\}$ of $\left\{x_{n}\right\}$ such that $\left\{x_{n_{i}}\right\} \rightarrow p$ and $\left\{x_{n_{j}}\right\} \rightarrow q$, respectively. We first show that $p, q \in F(T)$. In fact, if $T p \neq p$, then by Opial's condition and (12), we obtain

$$
\begin{aligned}
\limsup _{i \rightarrow \infty}\left\|x_{n_{i}}-p\right\| & <\limsup _{i \rightarrow \infty}\left\|x_{n_{i}}-T p\right\| \\
& \leq \limsup _{i \rightarrow \infty}\left\{\left\|x_{n_{i}}-T x_{n_{i}}\right\|+\left\|T x_{n_{i}}-T p\right\|\right\} \\
& \leq \limsup _{i \rightarrow \infty}\left\|x_{n_{i}}-p\right\| .
\end{aligned}
$$

This is a contradiction. Hence we obtain $T p=p$. Similarly, we obtain $q \in F(T)$. Next, as in the proof of (i) in Theorem 3.1, $\left\{x_{n}\right\}$ converges weakly to $p \in F(T)$.

Theorem 3.3 Let $H$ be a Hilbert space and $C$ be a nonempty, closed, and convex subset of $H$, and let $S, T$ be two quasi-nonexpansive mappings of $C$ into itself satisfying condition $\mathbf{A}$ with $\mathbf{F}=F(S) \cap F(T) \neq \emptyset$. Suppose that, for any $x_{1}$ in $C,\left\{x_{n}\right\}$ is defined by (1), where $\left\{\alpha_{n}\right\}$ and $\left\{\beta_{n}\right\}$ are chosen so that $\liminf _{n \rightarrow \infty} \alpha_{n}\left(1-\alpha_{n}\right)>0$ 
and $\liminf _{n \rightarrow \infty} \beta_{n}\left(1-\beta_{n}\right)>0$. Then $\left\{x_{n}\right\}$ converges strongly to a common fixed point of $S$ and $T$.

Proof As in the proof of (ii) in Theorem 3.1, we obtain

$$
\lim _{n \rightarrow \infty}\left\|T x_{n}-x_{n}\right\|=\lim _{n \rightarrow \infty}\left\|S x_{n}-x_{n}\right\|=0 .
$$

By using Condition A, we obtain

$$
f\left(d\left(x_{n}, \mathbf{F}\right)\right) \leq \frac{1}{2}\left\{\left\|T x_{n}-x_{n}\right\|+\left\|S x_{n}-x_{n}\right\|\right\}
$$

for all $n \geq 1$. As in the proof of Theorem 3.1,

$$
\left\|x_{n+1}-z\right\| \leq\left\|x_{n}-z\right\|,
$$

for any $z \in \mathbf{F}$. Taking the infimum over all $z \in \mathbf{F}$ on both sides, we see that $\lim _{n \rightarrow \infty} d\left(x_{n}, \mathbf{F}\right)(\equiv k)$ exists. We first claim that $\lim _{n \rightarrow \infty} d\left(x_{n}, \mathbf{F}\right)=0$. In fact, assume that $k=\lim _{n \rightarrow \infty} d\left(x_{n}, \mathbf{F}\right)>0$. Then we can choose $n_{0} \in N$ such that $0<\frac{k}{2}<$ $d\left(x_{n}, \mathbf{F}\right)$ for all $n \geq n_{0}$. By using Condition $\mathbf{A}$ and (13), we obtain

$$
0<f\left(\frac{k}{2}\right) \leq f\left(d\left(x_{n}, \mathbf{F}\right)\right) \leq \frac{1}{2}\left\{\left\|T x_{n}-x_{n}\right\|+\left\|S x_{n}-x_{n}\right\|\right\} \rightarrow 0
$$

as $n \rightarrow \infty$. This is a contradiction. So, we obtain $k=0$. Next, we claim that $\left\{x_{n}\right\}$ is a Cauchy sequence. Let $\epsilon>0$ be given. Since $\lim _{n \rightarrow \infty} d\left(x_{n}, \mathbf{F}\right)=0$, there exists $n_{0} \in N$ such that for all $n \geq n_{0}$, we obtain

$$
d\left(x_{n}, \mathbf{F}\right)<\frac{\epsilon}{2} .
$$

Let $n, m \geq n_{0}$ and $p \in \mathbf{F}$. Then, by (14), we obtain

$$
\begin{aligned}
\left\|x_{n}-x_{m}\right\| & \leq\left\|x_{n}-p\right\|+\left\|x_{m}-p\right\| \\
& \leq 2\left[\left\|x_{n_{0}}-p\right\|\right] .
\end{aligned}
$$

Taking the infimum over all $p \in \mathbf{F}$ on both sides and by (15), we obtain

$$
\left\|x_{n}-x_{m}\right\| \leq 2\left[d\left(x_{n_{0}}, \mathbf{F}\right)\right]<\epsilon
$$

for all $n, m \geq n_{0}$. This implies that $\left\{x_{n}\right\}$ is a Cauchy sequence. Let $\lim _{n \rightarrow \infty} x_{n}=q$. Since $\mathbf{F}$ is closed, we obtain $q \in \mathbf{F}$. Hence, $\left\{x_{n}\right\}$ converges strongly to a common fixed point of $S$ and $T$.

The following is an example of a quasi-nonexpansive mapping which is not nonexpansive mapping.

Example 3.1 [15] Let $\mathbb{R}$ denote the reals with the usual norm and $C=[-\pi, \pi]$. Let $T: C \rightarrow C$ be defined by

$$
T x=x \cos x
$$

for all $x \in C$. 
The following is an example of a quasi-nonexpansive mapping which are not a nonspreading mapping and a nonexpansive mapping.

Example 3.2 Let $\mathbb{R}$ denote the reals with the usual norm and $C=[0,2]$. Let $T: C \rightarrow$ $C$ be defined by

$$
T x=\frac{1}{3}\left(x^{2}+2\right)
$$

for all $x \in C$, where $x+2 \leq 3, x \in[0,1]$ and $x+2=3, x>1$. Clearly $F(T)=\{1,2\}$. $T$ is a quasi-nonexpansive mapping since if $x \in[0,2]$ and $z=1$, then

$$
|T x-z|=|T x-1|=\left|\frac{1}{3}\left(x^{2}+2\right)-1\right|=\frac{1}{3}\left|x^{2}-1\right| \leq|x-z| .
$$

If $x \in[0,2]$ and $z=2$, then

$$
|T x-z|=|T x-2|=\left|\frac{1}{3}\left(x^{2}+2\right)-2\right|=\frac{1}{3}\left|x^{2}-4\right| \leq|x-z| .
$$

But it is not a nonspreading mapping. In fact, if we take $x=2$ and $y=\sqrt{2}$, then we obtain $|T x-T y|^{2}=0.444,|x-y|^{2}=0.343,2\langle x-T x, y-T y\rangle=0$, and thus

$$
|T x-T y|^{2}=0.444>0.343=|x-y|^{2}+2\langle x-T x, y-T y\rangle .
$$

Also, it is not a nonexpansive mapping. In fact, if we take $x=2$ and $y=\sqrt{2}$, then we obtain

$$
|T x-T y|=0.666>0.586=|x-y| .
$$

\section{Concluding Remarks}

Our results, Theorems 3.1 and 3.2, carry over Theorem 4.1 of Iemoto and Takahashi to quasi-nonexpansive mappings. We study the strong convergence of the Moudafi's iterative scheme under the assumption that two quasi-nonexpansive self-mappings satisfying condition A. We give an example of a quasi-nonexpansive mapping which are not a nonspreading mapping and a nonexpansive mapping.

Open Access This article is distributed under the terms of the Creative Commons Attribution Noncommercial License which permits any noncommercial use, distribution, and reproduction in any medium, provided the original author(s) and source are credited.

\section{References}

1. Mann, W.R.: Mean value methods in iteration. Proc. Am. Math. Soc. 4, 506-510 (1953)

2. Reich, S.: Weak convergence theorems for nonexpansive mappings in Banach spaces. J. Math. Anal. Appl. 67, 274-276 (1979) 
3. Wittmann, R.: Approximation of fixed points of nonexpansive mappings. Arch. Math. 58, 486-491 (1992)

4. Tan, K.K., Xu, H.K.: Approximating fixed points of nonexpansive mappings by the Ishikawa Iteration process. J. Math. Anal. Appl. 178, 301-308 (1993)

5. Takahashi, W., Kim, G.E.: Approximating fixed points of nonexpansive mappings in Banach spaces. Math. Jpn. 48, 1-9 (1998)

6. Iemoto, S., Takahashi, W.: Approximating common fixed points of nonexpansive mappings and nonspreading mappings in a Hilbert space. Nonlinear Anal. 71(12), 2082-2089 (2009)

7. Moudafi, A.: Krasnoselski-Mann iteration for hierarchical fixed point problems. Inverse Probl. 23, 1635-1640 (2007)

8. Takahashi, W.: Introduction to Nonlinear and Convex Analysis. Yokohama Publishers, Yokohama (2005)

9. Browder, F.E.: Semicontractive and semiaccretive nonlinear mappings in Banach spaces. Bull. Am. Math. Soc. 74, 660-665 (1968)

10. Opial, Z.: Weak convergence of the sequence of successive approximations for nonexpansive mappings. Bull. Am. Math. Soc. 73, 591-597 (1967)

11. Khan, S.H., Fukhar-ud-din, H.: Weak and strong convergence of a scheme with errors for two nonexpansive mappings. Nonlinear Anal. 61, 1295-1301 (2005)

12. Senter, H.F., Dotson, W.G.: Approximating fixed points of nonexpansive mappings. Proc. Am. Math. Soc. 44, 375-380 (1974)

13. Groetsch, C.W.: A note on segmenting Mann iterates. J. Math. Anal. Appl. 40, 369-372 (1972)

14. Takahashi, W.: Nonlinear Functional Analysis. Kindaikagaku, Tokyo (1988) (Japanese)

15. Kim, G.E.: Convergence theorems for quasi-nonexpansive mappings in Banach spaces (submitted) 